\title{
Antidepressant drug use and future diabetes risk
}

\author{
M. Kivimäki • G. D. Batty
}

Received: 5 September 2011 / Accepted: 4 October 2011 /Published online: 29 October 2011

(C) The Author(s) 2011. This article is published with open access at Springerlink.com

Keywords Adverse drug effects · Antidepressants . Depression · Diabetes mellitus

\section{Abbreviations \\ FDA Food and Drug Administration \\ RCT Randomised controlled trial}

Debate around the side effects of antidepressants, one of the most prescribed groups of drugs worldwide [1], continues. First there was a concern that antidepressant medication may, with rather tragic irony, increase suicide risk. Then, meta-analyses suggested that there was no significant benefit of antidepressants over placebo in treating patients with minor depression [2]. The most recent controversy relates to a series of studies, including one in the present issue of Diabetologia, suggesting that long-term use of antidepressants might predispose individuals to type 2 diabetes [3-5].

Given the large number of existing randomised controlled trials (RCTs) on antidepressants and the fact that antidepressants are prescription drugs approved by governmental authorities in all Western countries, how is it possible that all major side effects have not already been ruled out and that efficacy still remains uncertain? One reason relates to publication bias. In 2008, Turner and colleagues analysed 74 RCTs approved by the US Food and

\footnotetext{
M. Kivimäki $(\bowtie) \cdot$ G. D. Batty

Department of Epidemiology and Public Health,

University College London,

1-19 Torrington Place,

WC1E 6BT London, UK

e-mail: m.kivimaki@ucl.ac.uk
}

Drug Administration (FDA) and found that a total of 37 of the 38 RCTs regarded by this agency as having positive results were published whereas $36 \mathrm{RCTs}$ viewed by the FDA as having negative or questionable results were, with three exceptions, either unpublished or published in a way that conveyed a positive outcome [6]. A 2010 review of four meta-analyses of efficacy RCTs submitted to the FDA confirmed this profound publication bias and additionally identified a new source of potential error [7]. The authors of the review noticed that in the Sequenced Treatment Alternatives to Relieve Depression (STAR*D) study, the largest antidepressant effectiveness trial ever conducted, there was an apparent progressively increasing dropout rate across each study phase [7]. If this dropout was selectiveand for this study it's unknown whether it was-it could seriously bias the findings.

Given these concerns, combined with the fact that diabetes is considerably under-diagnosed, it seems possible that diabetogenic side effects could have gone unnoticed in RCTs. This possibility is supported by observational data based on medical records showing that long-term antidepressant medication use is associated with an up to twofold increased risk for type 2 diabetes [3-5]. However, as with all study designs, observational data, particularly those in register-based studies, also have their methodological shortcomings. One is that the observed association can reflect common antecedent causes, such as weight gain, socioeconomic circumstances and lifestyle factors; that is, confounding factors that have not been measured, and have therefore not been taken into account. By contrast, investigator-led studies are typically better characterised.

An exemplar appears in this issue of Diabetologia, in which Pan and colleagues present new analyses based on three large well-known US datasets: the Nurses' Health 
Studies I and II and the Health Professionals Follow-up Study, a total of 139,000 women and 30,000 men [8]. Besides large sample sizes, these observational studies include measurements of lifetime history of depression, current depressive symptoms, and demographic, lifestyle and anthropometric data. During over 1.6 million personyears of follow-up, Pan et al. documented a total of 6,641 new cases of type 2 diabetes and found that self-reported antidepressant use was associated with an approximately $10-30 \%$ excess risk of developing diabetes. Importantly, this association was robust to adjustment for age, ethnicity, marital and living status, smoking habits, alcohol intake, physical activity, family history of diabetes, diet, body mass index and major comorbidities (e.g. hypertension and dyslipidaemia) and their treatments. In women, oral contraceptive use, menopausal status and hormone use were also taken into account. Furthermore, to allow for changes in antidepressant use and levels of covariates, the authors treated all these variables as time-dependent by entering in the equation data from each 2-4 year update. The study was therefore able to control for a much more comprehensive range of potential confounding factors and causes common to both antidepressant use/depression and diabetes than any of the previous observational investigations.

This evidence should be placed into context. First, some observational studies have not found a link between antidepressant use and diabetes $[9,10]$, raising the possibility that any positive association might be a chance finding. This seems unlikely, however, as the studies reporting a null finding have typically not assessed antidepressant use with repeated measurements and therefore may have missed any effects of long-term antidepressant use. Second, safety issues have been examined in registered RCTs of antidepressants and no clear evidence of increased diabetes risk has been documented. However, as the follow-up periods in trials are relatively short (typically 12 months rather than several years), these data do not necessarily rule out the possibility of an elevated risk of a condition like diabetes, which, like many chronic diseases, may have a long induction period. Third, it is also the case that depression per se, rather than antidepressant drugs themselves, could be elevating diabetes rates. A thorough investigation of this issue would require dissecting the effects of depression and antidepressant medication on diabetes risk; such data are currently lacking.

An important challenge to investigating the potential link between antidepressant drug therapy and future diabetes risk concerns the assessment of diabetes. By using self-reports, Pan et al. [8], in keeping with many other investigators [3-5], have measured only diagnosed diabetes. Failure to capture undiagnosed diabetes is clearly a potential source of error because it raises the possibility of detection bias as an alternative explanation for the observed associations. That is, a diagnosis of depression requiring pharmacological treatment might increase the likelihood of being diagnosed with other medical conditions such as diabetes, artificially creating a link between the two. There are ways of addressing this concern. One is to examine whether antidepressant use is associated with undiagnosed diabetes. If the relationship between antidepressant use and diabetes is truly causal, then antidepressant use should be associated with diabetes irrespective of the method of detection. In a related point, antidepressant use should also increase blood glucose levels among those participants who remain non-diabetic during study follow-up.

Currently, evidence regarding the existence of detection bias is mixed. In the Whitehall II study of British civil servants, exposure to antidepressant medication was monitored by repeated clinical interviews and undiagnosed diabetes was screened using an oral glucose tolerance test in four examinations over an 18 year follow-up period [11]. In this study, antidepressant use was associated with self-reported diagnosed diabetes but not with undiagnosed diabetes, higher fasting plasma glucose levels or increasing glucose levels over time [11]. These findings are consistent with the indication bias explanation and suggest that the antidepressant-diabetes association is spurious. By contrast, the Diabetes Prevention Program trial, which also used an oral glucose tolerance test to detect diabetes, suggests that the link may be 'real' [12]. The study recruited people at high risk of diabetes (overweight and elevated blood glucose levels) who did not meet the criteria for a diagnosis of diabetes. Participants were randomly assigned to receive lifestyle intervention, glucose-lowering medication (metformin) or placebo [12]. The authors found that in the lifestyle and placebo groups, participants who were consistently on antidepressants during the study period were about twice as likely as non-users to develop diabetes (no such pattern was seen in the metformin group).

Taking these results together, at this stage we cannot conclude that antidepressants increase the risk of diabetes, but we have no strong grounds to exclude this possibility either. Diabetes is a serious chronic disease that, because it can cause a catastrophic failure of function in an array of vital organs, especially the eyes, kidneys, nerves, heart, and blood vessels, is the seventh leading cause of death in the US [13]. If antidepressants really do have important diabetogenic side effects, this would potentially have major implications for the treatment of depression. In light of the new findings reported in this issue of Diabetologia, a closer evaluation of the safety and cost-benefit of antidepressants is certainly needed. 
Duality of interest The authors declare that there is no duality of interest associated with this manuscript.

Open Access This article is distributed under the terms of the Creative Commons Attribution Noncommercial License which permits any noncommercial use, distribution, and reproduction in any medium, provided the original author(s) and source are credited.

\section{References}

1. Olfson M, Marcus SC (2009) National patterns in antidepressant medication treatment. Arch Gen Psychiatry 66:848-856

2. Barbui C, Cipriani A, Patel V, Ayuso-Mateos JL, van Ommeren M (2011) Efficacy of antidepressants and benzodiazepines in minor depression: systematic review and meta-analysis. Br J Psychiatry 198:11-16

3. Brown LC, Majumdar SR, Johnson JA (2008) Type of antidepressant therapy and risk of type 2 diabetes in people with depression. Diab Res Clin Pract 79:61-67

4. Andersohn F, Schade R, Suissa S, Garbe E (2009) Long-term use of antidepressants for depressive disorders and the risk of diabetes mellitus. Am J Psychiatry 166:591-598

5. Kivimäki M, Hamer M, Batty GD et al (2010) Antidepressant medication use, weight gain and risk of type 2 diabetes mellitus: a population-based study. Diabetes Care 33:2611-2616
6. Turner EH, Matthews AM, Linardatos E, Tell RA, Rosenthal R (2008) Selective publication of antidepressant trials and its influence on apparent efficacy. N Engl J Med 358:252-260

7. Pigott HE, Leventhal AM, Alter GS, Boren JJ (2010) Efficacy and effectiveness of antidepressants: current status of research. Psychother Psychosom 79:267-279

8. Pan A, Sun Q, Okereke OI et al (2011) Use of antidepressant medication and risk of type 2 diabetes: results from three cohorts of US adults. Diabetologia. doi:10.1007/s00125-011-2268-49

9. Campayo A, de Jonge P, Roy JF, Saz P et al (2010) Depressive disorder and incident diabetes mellitus: the effect of characteristics of depression. Am J Psychiatry 167:580-588

10. Knol MJ, Geerlings MI, Egberts AC, Gorter KJ, Grobbee DE, Heerdink ER (2007) No increased incidence of diabetes in antidepressant users. Int Clin Psychopharmacol 22:382386

11. Kivimäki M, Batty GD, Jokela M et al (2011) Antidepressant medication use and risk of hyperglycaemia and diabetes mellitus-a non-causal association? Biol Psychiatry. doi:10.1016/j.biopsych.2011.07.008

12. Rubin RR, Ma Y, Peyrot M et al (2010) Antidepressant medicine use and risk of developing diabetes during the Diabetes Prevention Program and Diabetes Prevention Program Outcomes Study. Diabetes Care 33:2549-2551

13. Centers for Disease Control and Prevention (2011) National Diabetes Fact Sheet: national estimates and general information on diabetes and prediabetes in the United States, 2011. US Department of Health and Human Services, Centers for Disease Control and Prevention, Atlanta 